\title{
Sustained Maintenance of Somatotopic Information in Brain Regions Recruited by Tactile Working Memory
}

\author{
Tobias Katus, ${ }^{1,2}$ Matthias M. Müller, ${ }^{2}$ and Martin Eimer ${ }^{1}$ \\ ${ }^{1}$ Department of Psychology, Birkbeck College, University of London, London WC1E 7HX, United Kingdom, and ${ }^{2}$ Institut für Psychology, Universität \\ Leipzig, 04109 Leipzig, Germany
}

To adaptively guide ongoing behavior, representations in working memory (WM) often have to be modified in line with changing task demands. We used event-related potentials (ERPs) to demonstrate that tactile WM representations are stored in modality-specific cortical regions, that the goal-directed modulation of these representations is mediated through hemispheric-specific activation of somatosensory areas, and that the rehearsal of somatotopic coordinates in memory is accomplished by modality-specific spatial attention mechanisms. Participants encoded two tactile sample stimuli presented simultaneously to the left and right hands, before visual retro-cues indicated which of these stimuli had to be retained to be matched with a subsequent test stimulus on the same hand. Retro-cues triggered a sustained tactile contralateral delay activity component with a scalp topography over somatosensory cortex contralateral to the cued hand. Early somatosensory ERP components to task-irrelevant probe stimuli (that were presented after the retro-cues) and to subsequent test stimuli were enhanced when these stimuli appeared at the currently memorized location relative to other locations on the cued hand, demonstrating that a precise focus of spatial attention was established during the selective maintenance of tactile events in WM. These effects were observed regardless of whether participants performed the matching task with uncrossed or crossed hands, indicating that WM representations in this task were based on somatotopic rather than allocentric spatial coordinates. In conclusion, spatial rehearsal in tactile WM operates within somatotopically organized sensory brain areas that have been recruited for information storage.

Key words: electroencephalography; event-related potentials; selective attention; somatosensation; working memory

\section{Introduction}

In the year of its 40th birthday, the most influential structural account of working memory (WM; Baddeley and Hitch, 1974) still lacks a distinct memory module for the retention of tactile information. Recently, Baddeley (2012) hypothesized that tactile information may converge onto the storage component for visual information, the visuospatial sketchpad. Storing tactile locations in a nontactile format would require the recoding of anatomically referenced (somatotopic) coordinates into a modality-independent (allocentric) reference frame. Perceptual studies that used purely tactile (Soto-Faraco and Azañón, 2013) or cross-modal tactilevisual paradigms (Azañón and Soto-Faraco, 2008) revealed evidence for such a remapping process, which is automatically

\footnotetext{
Received Aug. 22, 2014; revised Nov. 16, 2014; accepted Nov. 19, 2014.

Author contributions: T.K. designed research; T.K. performed research; T.K. analyzed data; T.K., M.M.M., and M.E. wrote the paper.

This work was supported by Deutsche Forschungsgemeinschaft Grants KA 3843/1-1 and MU 972/15-1 and by a grant from the Economic and Social Research Council, United Kingdom. We thank Anna Grubert and John Towler for constructive comments on this manuscript and Renate Zahn for assistance in data acquisition.

The authors declare no competing financial interests.

This article is freely available online through the J Neurosci Author Open Choice option.

Correspondence should be addressed to Matthias M. Müller, Institut für Psychologie, Universität Leipzig, Neumarkt 9-19, 04109 Leipzig, Germany. E-mail: m.mueller@rz.uni-leipzig.de.

DOI:10.1523/JNEUROSCI.3535-14.2015

Copyright $\odot 2015$ Katus et al.

This is an Open Access article distributed under the terms of the Creative Commons Attribution License (http://creativecommons.org/licenses/by/3.0), which permits unrestricted use, distribution and reproduction in any medium provided that the original work is properly attributed.
}

triggered by tactile stimulation (Azañón et al., 2010). Remapping computes allocentric coordinates for stimuli based on their locations on the skin and the current posture of the body.

Sensory recruitment accounts of WM (Pasternak and Greenlee, 2005; D'Esposito, 2007) assume that somatosensory brain areas store locations in a modality-specific (somatotopic) format. Visual and tactile WM rely on distributed neural networks that include prefrontal cortex (PFC; Romo and Salinas, 2003; Kostopoulos et al., 2007) and modality-specific perceptual areas (Zhou and Fuster, 1996; Harrison and Tong, 2009). According to the sensory recruitment model, memory traces are stored in sensory brain areas, whereas PFC performs top-down control functions that guide the goal-dependent modulation of WM representations (Lepsien and Nobre, 2006; Postle, 2006). Support for sensory recruitment comes from event-related potential (ERP) studies of visual WM that identified a sustained negativity [contralateral delay activity (CDA)] at posterior electrodes contralateral to the side of memorized objects during the retention period (Vogel and Machizawa, 2004). A tactile CDA (tCDA) component was recently found over somatosensory areas contralateral to the hand where tactile information was memorized (Katus et al., 2014b). The tCDA component is sensitive to tactile memory load and individual differences in working memory capacity and indexes the sustained spatial rehearsal of tactile information.

The present experiment investigated whether spatially selective maintenance processes in tactile WM operate within an allo- 

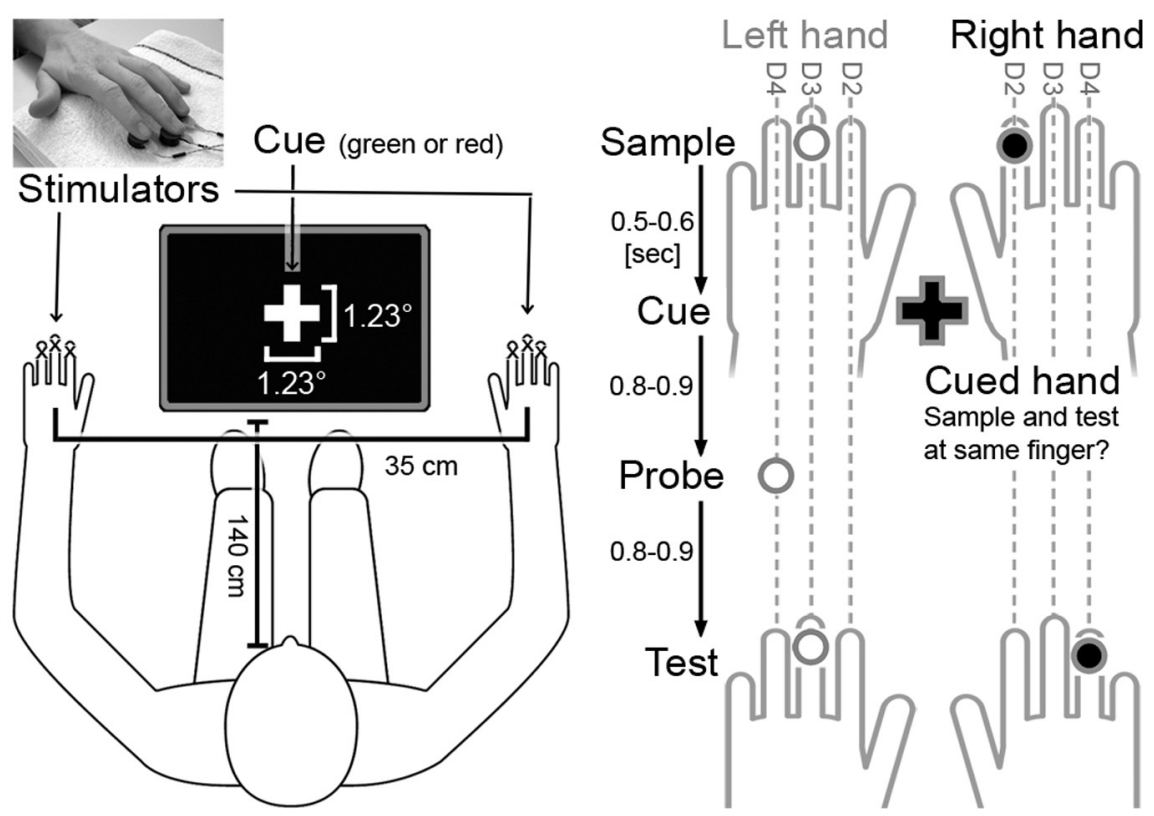

Figure 1. Stimulus material and task design. Top left, Six tactile stimulators were attached to the distal phalanges of the index (D2), middle (D3), and ring (D4) fingers of the left and right hands. Two sample stimuli were presented simultaneously to one finger of the left and right hands. A visual retro-cue signaled which of these two stimuli had to be retained and compared with the location of a subsequent test pulse at the same hand. The cue was followed by a task-irrelevant unilateral tactile probe and task-relevant bilateral test stimuli. In the example (shown for a block with uncrossed hand posture), the retro-cue indicates that the sample stimulus on the right hand has to be memorized, and the test stimulus presented to this hand does not match the location of the sample.

centric or somatotopic reference frame. Participants encoded two tactile sample stimuli simultaneously presented to the left and right hands, before a visual retro-cue indicated which of these stimuli had to be matched with a subsequent tactile test stimulus (Fig. 1). Retro-cues were presented between 500 and $600 \mathrm{~ms}$ after the samples to allow for the remapping of somatotopic coordinates into allocentric space (cf. Azañón and Soto-Faraco, 2008). We contrasted spatial rehearsal of cued tactile information in WM (indexed by the tCDA component) when allocentric and somatotopic coordinates were aligned (uncrossed hands) or in conflict (crossed hands). If rehearsal is based on modality-specific somatotopic WM representations, hand posture should not influence tCDAs elicited by retro-cues. If it operates within a modalityindependent allocentric reference frame, tCDA components elicited by retro-cues should reverse polarity when hand posture is changed. To examine the role of focal spatial attention during spatially selective tactile WM maintenance and its link to hand posture, we also measured attentional modulations of somatosensory ERPs to task-irrelevant tactile probes and to the subsequent test stimuli.

\section{Materials and Methods}

Participants. Twenty neurologically unimpaired adult participants were tested (14 female; mean age, 23 years; range, $19-34$ years). All participants were right-handed, gave informed written consent before testing, and received money or course credit. The study was conducted in accordance with the Declaration of Helsinki and approved by the local ethical committee.

Stimuli and task design. Participants were seated in a dimly lit recording chamber with their hands covered from sight. Six tactile stimulators were attached to the distal phalanges of the index, middle, and ring fingers of the left and right hands. Stimulators were driven by a dualchannel amplifier controlled by custom-written MATLAB scripts (The MathWorks) running on a Windows XP computer. White noise masked sounds produced by tactile stimulation. All tactile stimuli (sample, probe, and test stimuli) were mechanical pulses (duration, $5 \mathrm{~ms}$; intensity, $0.37 \mathrm{~N}$ ). Visual stimuli (retro-cues) were centrally presented on a monitor (Fig. 1).

Each trial started with the presentation of a bilateral pair of tactile sample stimuli, which was followed by a visual retro-cue, a unilateral tactile probe stimulus, and a pair of bilateral tactile test stimuli (Fig. 1). Two sample pulses (one per hand) were simultaneously presented to one randomly selected finger of the left hand and one randomly determined finger of the right hand. The visual retro-cue was presented at a randomly determined time point between 500 and $600 \mathrm{~ms}$ after sample stimulus onset on the computer monitor by changing the white fixation cross to green or red (duration of color change, $150 \mathrm{~ms}$ ). The color of the retro-cue indicated whether the tactile memory matching task had to be performed for the left or right hand. The color-hand assignment remained constant for each participant and was counterbalanced across participants. A unilateral taskirrelevant tactile probe stimulus was presented between 800 and $900 \mathrm{~ms}$ after the onset of the retro-cue, with equal probability to one of the six possible tactile locations on the left and right hands. At 800-900 ms after the probe, a bilateral pair of tactile test stimuli was presented to the left and right hands. On match trials, the test pulse on the cued (relevant) hand was presented to the same finger that had received the sample pulse. On mismatch trials, the test pulse on the cued hand was delivered to one of the two fingers that were not stimulated by the sample pulse. Match and mismatch trials were equally likely $(50 \%)$. The location of the test pulse on the uncued (irrelevant) hand was determined in a fashion analogous to the cued hand. Because the randomization was performed for each hand separately, locations of test pulses were not correlated between the cued and uncued hands.

Participants were instructed to initially encode both tactile sample stimuli and then to decide on each trial whether sample and test stimulus locations on the hand indicated by the retro-cue matched or did not match. They signaled a match or mismatch with a vocal response ("a" for match, "e" for mismatch) recorded by a microphone. The response period (duration, $2600 \mathrm{~ms}$ ) was indicated by a question mark that appeared on the monitor $400 \mathrm{~ms}$ after test stimulus onset. In alternating blocks, this task was performed with uncrossed or crossed hands. Hand posture in the first experimental block was counterbalanced across participants.

The experiment consisted of 10 blocks. Each block contained 56 trials and took about $5 \mathrm{~min}$ to complete. Performance feedback was given after each block. Participants performed an equal number of training trials with uncrossed and crossed hand posture (80-120 trials for each posture, dependent on participants' performance during training). They were asked to avoid head and arm movements and to maintain gaze fixation during EEG recordings. Task instructions emphasized accuracy over speed.

Data recording and analysis. EEG data, sampled at $256 \mathrm{~Hz}$ using a Biosemi ActiveTwo amplifier, were recorded from $64 \mathrm{Ag} / \mathrm{AgCl}$ active electrodes at standard locations of the international 10-10 system. A bipolar outer canthus montage [horizontal electro-oculogram (HEOG)] monitored lateral eye movements; a montage below and above the right eye (vertical electro-oculogram) monitored vertical eye movements and blinks. Continuous EEG data were referenced to the arithmetic mean of both mastoids and submitted to a digital $40 \mathrm{~Hz}$ low-pass finite impulse response filter (Blackman window, filter order 666). ERP epochs were computed for the $1800 \mathrm{~ms}$ interval after the visual retro-cue, for the 300 $\mathrm{ms}$ interval after the tactile probe stimulus, and for the $500 \mathrm{~ms}$ interval 
after the tactile test stimuli, and these epochs were corrected relative to the $100 \mathrm{~ms}$ prestimulus baselines.

Blind source separation of EEG data was performed with the Independent Component Analysis (ICA) algorithm implemented in the EEGLab toolbox (Delorme and Makeig, 2004). Independent components related to eye blinks, vertical and horizontal eye movements were identified by visual inspection and subtracted from EEG sets (Delorme et al., 2007). On average, $2.3 \%$ of epochs contained lateral eye movements, as indicated by a differential step function running on the bipolarized HEOG (step width, $100 \mathrm{~ms}$; threshold, $24 \mu \mathrm{V}$ ). None of these epochs was marked for rejection by the same function after ICA was used to correct for lateral eye movement artifacts. Epochs entered FASTER (Fully Automated Statistical Thresholding for EEG Artifact Rejection; Nolan et al., 2010) and were visually rechecked. Approximately $90.7 \%$ of all epochs to retro-cues, $92.6 \%$ of all epochs to probes, and $92.2 \%$ of test stimulus epochs were retained for statistical analysis. Analyses included trials with correct and incorrect responses to maximize the signal-tonoise ratio. The exclusion of incorrect trials yielded an identical pattern of results for all measures of interest.

ERP waveforms were separately averaged across six lateral electrodes (FC3/4, FC5/6, $\mathrm{C} 3 / 4, \mathrm{C} 5 / 6, \mathrm{CP} 3 / 4, \mathrm{CP} 5 / 6)$ over somatosensory brain areas contralateral and ipsilateral to the currently task-relevant hand. Statistical tests were conducted on mean amplitudes for the time windows of respective ERP components (see below). Data shown in spline-interpolated topographical voltage maps were collapsed across trials in which the left or right hand was task relevant by flipping electrode coordinates in trials with left-hand cues over the midline. Error bars in graphs showing difference values reflect $95 \%$ confidence intervals that were calculated for each condition by $t$ tests against zero (i.e., no difference). Statistical significance of difference values is symbolized by asterisks $\left({ }^{*} p<0.05 ;{ }^{* *} p<0.01 ;{ }^{* *} p<0.001\right)$ and is marked by error bars that do not overlap with the zero axis.

Onset latency differences between tCDA components in blocks with uncrossed versus crossed hands were assessed with a jackknife-based procedure (Miller et al., 1998). Twenty grand-average difference waves were computed for both hand posture conditions, each excluding one different participant from the original sample, and tCDA onset latency was defined as the point in time when each subsample difference wave reached an absolute onset criterion of $-0.3 \mu \mathrm{V}$. Onset latency differences between the two conditions were tested with a two-tailed $t$ test, with the $t$ value corrected according to the formulas described by Miller et al. (1998).

\section{Results}

\section{Delay period activity evoked by retro-cues}

Visual retro-cues were presented 500-600 ms after the tactile sample set and indicated whether the left- or right-hand sample pulse had to be selectively maintained. To determine whether selective attention to one of the two stimuli in tactile working memory would trigger sustained maintenance-related delay period activity over contralateral somatosensory cortex, difference waveforms were calculated by subtracting ERP traces recorded ipsilateral to the cued hand from contralateral ERPs, and statistical analyses were conducted on mean difference amplitudes measured for the tCDA component time window (from $350 \mathrm{~ms}$ after retro-cue onset until $1600 \mathrm{~ms}$, i.e., the onset of bilateral test pulses) across the six lateral central electrode pairs. The presence of reliable lateralized effects is marked by difference values that significantly deviate from zero.

Retro-cues elicited a sustained negativity (tCDA component) over somatosensory scalp regions contralateral to the relevant (cued) hand. As shown in Figure 2, tCDA components emerged around $350 \mathrm{~ms}$ after retro-cue onset and remained present throughout the remaining delay period. Statistically reliable tCDAs were present in blocks with uncrossed $\left(t_{(19)}=-6.439\right.$, $\left.p<10^{-5}\right)$ and crossed $\left(t_{(19)}=-7.166, p<10^{-6}\right)$ hand posture. The size of the tCDA component did not differ between blocks with uncrossed and crossed hand posture $(p>0.7)$, demonstrating that the maintenance-related activity reflected by this component was determined by anatomical rather than allocentric coordinates of the memorized stimulus. However, as can be seen in Figure 2, the tCDA emerged slightly earlier when hands were uncrossed. An analysis of tCDA amplitudes during the 350-500 ms interval after retro-cue onset confirmed this observation. A reliable tCDA was already present during this early interval with uncrossed hand posture $\left(t_{(19)}=-3.654, p=0.002\right)$ but not with crossed hands $\left(t_{(19)}=-1.452, p=0.163\right)$. The delay of the tCDA with crossed hands was formally assessed with a jackknife-based procedure (Miller et al., 1998). The tCDA emerged $335 \mathrm{~ms}$ after retro-cue onset with uncrossed hands and $426 \mathrm{~ms}$ after cue onset with crossed hands. This onset latency difference of $91 \mathrm{~ms}$ was significant $\left(t_{(19)}=4.431, p<0.001\right.$; proportion of explained variance $\left.r^{2}=0.508\right)$.

\section{ERPs to tactile probe stimuli}

Figure $3 A$ shows ERPs elicited by tactile probes presented to the relevant hand at contralateral electrodes over somatosensory cor- 


\section{A Probe stimulus}
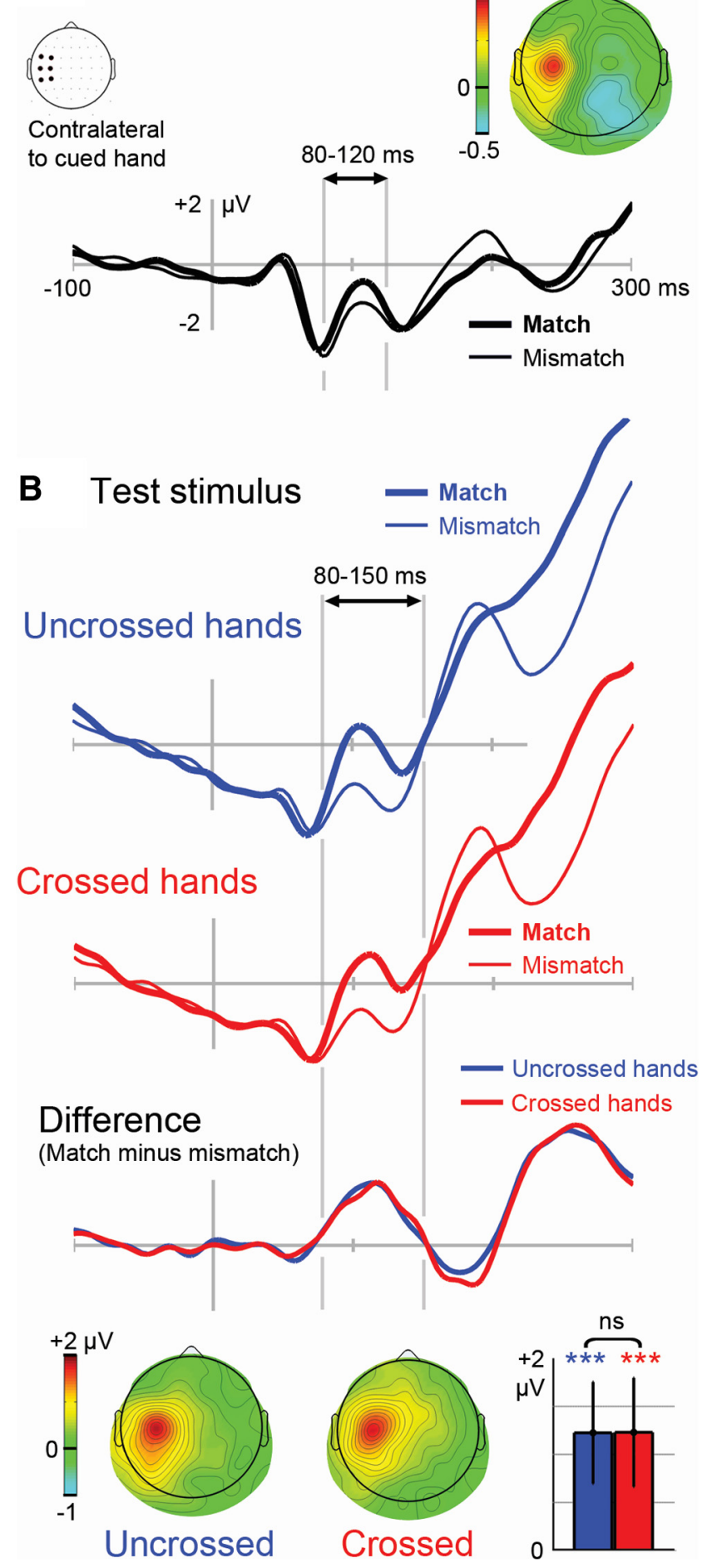

Figure 3. A, ERPs elicited by tactile probe stimuli delivered to the task-relevant hand at electrodes contralateral to this hand during the 300 ms interval after probe onset, collapsed across blocks with uncrossed and crossed hands. ERPs are shown separately for trials where a probe was presented at the same finger as the memorized sample stimulus (match trials) and trials where the probe was delivered to another finger of the same hand (mismatch trials). The somatosensory P100 component was enhanced on match trials, and the map shows the topographical distribution of this P100 modulation on the basis of difference waves obtained by subtracting ERP mean amplitudes measured in the P100 time range (80-120 ms after probe onset) on mismatch trials from ERPs on match trials. $\boldsymbol{B}$, ERPs elicited by bilateral tactile test stimuli at electrodes contralateral to the task-relevant hand during the $300 \mathrm{~ms}$ interval after test onset, on match trials where the test stimulus was presented at the same finger as the memorized sample stimulus and on mismatch trials where it was delivered to another finger of the tex, separately for probes presented to the finger that had received the to-be-memorized sample pulse and for probes presented to one of the fingers that were not stimulated by a sample pulse. The somatosensory P100 component was enhanced when probes were presented to the same finger that was stimulated by the preceding task-relevant sample pulse relative to probes presented to one of the other fingers of the same hand. A formal test of ERP mean amplitudes measured in a time window centered on the P100 component ( $80-120 \mathrm{~ms}$ after probe onset) obtained a reliable effect of probe location (memorized vs not memorized finger $)$ on $\mathrm{P} 100$ amplitude $\left(F_{(1,19)}=5.221, p=0.034\right)$. There was no significant main effect of hand posture (uncrossed vs crossed, $p>$ $0.4)$ and no reliable hand posture $X$ probe location interaction $(p>0.6)$.

\section{ERPs to tactile test stimuli}

After the retention period, tactile test stimuli to the cued taskrelevant hand were either delivered to the same finger where a sample had been presented (match trial, 50\%) or to a different finger (mismatch trial, 50\%). Figure $3 B$ shows ERPs triggered by test stimuli at electrodes recorded contralateral to the taskrelevant hand on match and mismatch trials, separately for blocks with uncrossed and crossed hand posture. Similar to observations for tactile probes, the somatosensory P100 component was enhanced on match trials where a test stimulus was delivered to the same finger that had received the memorized sample pulse relative to mismatch trials where a different finger of the same hand was stimulated at test. In contrast to the probe stimulus results, this attentional modulation of somatosensory ERPs extended beyond the P100 component and also modulated the subsequent N140 component. To assess the reliability of this effect, mean ERP amplitudes at central electrodes contralateral to the task-relevant hand were computed within a time window that overlapped with the P100 component and N140 components (80-150 ms after stimulus). Significant differences between match and mismatch trials were present both with uncrossed hands $\left(t_{(19)}=4.466, p<0.001\right)$ and with crossed hands $\left(t_{(19)}=\right.$ $\left.5.149, p<10^{-4}\right)$. The size of these attentional modulations did not differ between hand postures $(p>0.8)$.

\section{Behavioral performance}

Participants correctly reported a match or mismatch between tactile sample and test stimuli on the cued hand in $93.2 \%$ of all trials with uncrossed hands and in $86.0 \%$ of all trials with crossed hands. An analysis of sensitivity indices $\left(\mathrm{d}^{\prime}\right)$ by a two-way repeated-measures ANOVA with the factors cued hand (left, right) and hand posture (uncrossed, crossed) confirmed that this effect of hand posture was reliable $\left(F_{(1,19)}=26.335, p=10^{-4}\right)$. There was no significant main effect of cued hand and no hand posture $\times$ cued hand interaction.

\section{Discussion}

We examined spatial rehearsal in somatosensory brain areas using a tactile spatial WM task. Two bilaterally presented tactile

$\leftarrow$

same hand. ERPs are shown separately for blocks with uncrossed and crossed hands. The somatosensory P100 component was enhanced on match trials, and this attentional modulation overlapped with the subsequent N140 component. The topographical maps show the distribution of this effect during the $80-150$ ms interval after test stimulus onset. They were obtained by subtracting ERPs on mismatch trials from ERPs on match trials. Difference waves (match minus mismatch trials) show identical attentional modulations in blocks with uncrossed and crossed hands. Bar graphs show mean difference amplitudes at contralateral central electrodes. Error bars reflect $95 \%$ confidence intervals of difference values tested against zero. 
sample stimuli first had to be encoded in parallel before a visual retro-cue indicated which of these stimuli had to be selectively maintained. Because the location of the target stimulus was not known before the retro-cue was presented, WM initially contained neural representations of tactile stimuli on both hands, unbiased by top-down control, in the period between sample set and retro-cue. The retrospective cueing procedure served to delay spatial rehearsal in WM to allow for a complete remapping of the tactile sample set into an allocentric reference frame (cf. Azañón and Soto-Faraco, 2008). In different blocks, the memory task was performed with uncrossed and crossed hand posture to selectively manipulate allocentric stimulus coordinates, which depend on whether a stimulated limb is located at the left or right side of the body. Electrophysiological data revealed that spatially selective WM maintenance processes in somatosensory brain areas are not sensitive to manipulations of body posture. This demonstrates that spatial rehearsal of tactile information operates within the somatotopically organized spatial layout of tactile WM in sensory brain areas that have been recruited for information storage.

\section{Sustained rehearsal of task-relevant coordinates in somatotopic space}

Retro-cues triggered a tCDA component over somatosensory cortex contralateral to the cued hand in both hand posture conditions (Fig. 2) and resulted in attentional processing enhancements for tactile probes and test stimuli that were presented at to-be-retained locations (Fig. 3). A previous study (conducted with uncrossed hands) has shown that the tCDA component emerges during the maintenance of tactile information when there is no conflict between allocentric and somatotopic reference frames (Katus et al., 2014b). The presence of a tCDA, contralateral to the cued hand when these reference frames were misaligned (crossed hands; Fig. 2), indicates that the tCDA reflects the sustained engagement of modality-specific control mechanisms that modulate the content of tactile WM in a goaldirected fashion through the spatially selective biasing of somatotopic representations in tactile WM.

The fact that tCDA components were very similar in size when participants performed the task with crossed or uncrossed hands provides additional evidence that the memory representations that were maintained in a spatially selective fashion were not stored in an allocentric format. Crossing the hands induces a conflict between anatomical and allocentric spatial coordinates, which can impair tactile judgments (Yamamoto and Kitazawa, 2001) and the attentional selection of tactile events (Eimer et al., 2003). Despite the robust reduction in behavioral performance when hands were crossed, the onset of the tCDA component was only moderately delayed by $<100 \mathrm{~ms}$, suggesting that a conflict of reference frames has a relatively small effect on somatosensory processes involved in the storage and maintenance of tactile information.

\section{Spatial attention in tactile WM operates within a narrow focus of somatotopic space}

Whereas the presence of tCDA components in response to visual retro-cues indicates different activation levels for the contralateral versus ipsilateral hemispheres during retention, this hemispherical asymmetry in neural processing does not provide more detailed insights into the spatial specificity of attentional modulations. Retro-cues might have triggered a shift of spatial attention toward the relevant hand, resulting in a global enhancement of processing in somatosensory cortex contralateral to the mem- orized hand. Alternatively, they may have elicited a more spatially selective attentional facilitation of tactile processing at somatotopic locations that matched the position of the memorized finger. The ERP results obtained in response to unilateral taskirrelevant probe stimuli delivered to the cued hand provide clear evidence for a narrow focus of spatial attention during tactile WM maintenance. These probes appeared 800-900 ms after the retro-cue and triggered an attentional enhancement of the somatosensory P100 component when they were presented to the location that had previously received the tactile sample stimulus, relative to probes that were presented to a different finger of the same cued hand (Fig. 3A). We recently compared ERPs for tactile probe stimuli presented at the cued hand with probes at the uncued hand and found an attentional modulation of the N140 component, indicating that the allocation of spatial attention was biased toward the hand where a location is memorized during retention (Katus et al., 2012). The modulation of the somatosensory P100 component at scalp regions contralateral to the hand where stimuli were memorized in the present study demonstrates that during the selective maintenance of a tactile stimulus at a particular location on the cued hand, spatial attention was focused on the exact location of this stimulus in somatotopic space [see Eimer and Forster (2003) and Heed and Röder (2010) for similar ERP effects in perceptual tactile attention tasks].

The pattern of ERP results obtained in response to bilateral tactile test stimuli (Fig. 3B) confirmed this conclusion. P100 components triggered at electrodes contralateral to the cued hand were enhanced on trials where a test stimulus matched the exact location of the sample stimulus on this hand, relative to trials where test stimuli appeared at a nonmatching location. This attentional enhancement of ERPs to test stimuli was similar to the corresponding modulation of probe ERPs (Fig. 3A) and overlapped with the subsequent somatosensory N140 component. For these stimuli, ERP modulations were virtually identical in blocks with uncrossed and crossed hand posture (Fig. 3B, difference wave), again demonstrating that somatosensory encoding was guided by mnemonic content referenced to somatotopic rather than allocentric space.

It is well established that the rehearsal of tactile and visual information in WM is mediated by qualitatively similar strategies, which involve covert spatial attention shifts to memorized locations [vision (Awh et al., 2006) and touch (Katus et al., 2012)]. Since visual locations are referenced to retinotopic or spatiotopic rather than somatotopic space, the neural implementations of these attention-based rehearsal mechanisms are obviously different for visual versus tactile information. This is in line with the sensory recruitment model that assumes that the WM maintenance of information from different sensory modalities is implemented in separate modality-specific memory systems.

\section{No evidence for allocentric remapping during WM maintenance of tactile locations}

The bulk of computations for tactile remapping are performed between 70 and $190 \mathrm{~ms}$ after stimulus [Soto-Faraco and Azañón, 2013; see Azañón and Soto-Faraco (2008) for evidence that remapping may extend up to $360 \mathrm{~ms}$ after stimulus]. Although remapping is ongoing, the concurrent presence of somatotopic and allocentric codes between 100 and $140 \mathrm{~ms}$ after stimulus (Heed and Röder, 2010) interferes with the spatial selection of perceptually presented stimuli and leads to reduced attentional modulations of the P100 and N140 components if somatotopic and allocentric reference frames are misaligned (Eimer et al., 2003). No such interference effects on somatosensory ERP com- 
ponents were found here. These results seemingly suggest that our participants did not remap tactile stimuli to allocentric space, which would contradict previous findings that remapping occurs automatically in response to tactile stimulation (Gallace et al., 2008; Azañón et al., 2010). However, these studies also indicated that remapping can show a considerable degree of flexibility as the spatial reference frame adopted by participants critically depends on the specific demands of an experimental task. Such observations suggest that tactile processing is based on spatial representations that result from a weighted integration of somatotopic coordinates with postural information (de Haan et al., 2012). The dominance of somatotopic space during memory maintenance observed in the current experiment may thus reflect the high priority of somatotopic spatial coordinates in a task where successively presented tactile stimuli have to be spatially matched on the skin's surface. Future work needs to clarify the conditions under which allocentric coordinates and supramodal attentional control processes can affect spatially selective WM maintenance processes in somatosensory cortical regions.

\section{Theoretical perspectives}

Our findings contradict the assertion that tactile information is always automatically recoded into allocentric space and that this recoding is critical for the storage of tactile information within a nontactile memory system (e.g., the visuospatial sketchpad). They also highlight the need to include a dedicated storage component for somatosensory information within current structural models of WM (Baddeley, 2012). Our results support sensory recruitment accounts (Pasternak and Greenlee, 2005; Postle, 2006) that postulate that tactile WM emerges when selective attention is directed to memory traces, residing in somatosensory brain regions that were recruited for information storage. The information contained in this modality-specific, somatotopically organized storage system can be flexibly altered according to behavioral goals and is sustained by spatial rehearsal mechanisms that regulate the activation of tactile representations in somatosensory cortex. Spatial attention plays a central role as a rehearsal mechanism for visual (Awh et al., 2000) and tactile (Katus et al., 2014a) locations. Despite this apparent similarity across vision and touch, we show that spatial rehearsal of tactile information is mediated by distinct modality-specific control mechanisms that influence neural processing at the site where information is stored.

\section{References}

Awh E, Anllo-Vento L, Hillyard SA (2000) The role of spatial selective attention in working memory for locations: evidence from event-related potentials. J Cogn Neurosci 12:840-847. CrossRef Medline

Awh E, Vogel EK, Oh SH (2006) Interactions between attention and working memory. Neuroscience 139:201-208. CrossRef Medline

Azañón E, Soto-Faraco S (2008) Changing reference frames during the encoding of tactile events. Curr Biol 18:1044-1049. CrossRef Medline

Azañón E, Camacho K, Soto-Faraco S (2010) Tactile remapping beyond space. Eur J Neurosci 31:1858-1867. CrossRef Medline

Baddeley A (2012) Working memory: theories, models, and controversies. Annu Rev Psychol 63:1-29. CrossRef Medline

Baddeley AD, Hitch G (1974) Working memory. In: The psychology of learning and motivation: advances in research and theory, Vol 8 (Bower GH, ed), pp 47-89. New York: Academic.

de Haan AM, Anema HA, Dijkerman HC (2012) Fingers crossed! An investigation of somatotopic representations using spatial directional judgements. PLoS One 7:e45408. CrossRef Medline

Delorme A, Makeig S (2004) EEGLAB: an open source toolbox for analysis of single-trial EEG dynamics including independent component analysis. J Neurosci Methods 134:9-21. CrossRef Medline

Delorme A, Sejnowski T, Makeig S (2007) Enhanced detection of artifacts in EEG data using higher-order statistics and independent component analysis. Neuroimage 34:1443-1449. CrossRef Medline

D’Esposito M (2007) From cognitive to neural models of working memory. Philos Trans R Soc Lond B Biol Sci 362:761-772. CrossRef Medline

Eimer M, Forster B (2003) The spatial distribution of attentional selectivity in touch. Evidence from somatosensory ERP components. Clin Neurophysiol 114:1298-1306. CrossRef Medline

Eimer M, Forster B, Van Velzen J (2003) Anterior and posterior attentional control systems use different spatial reference frames. ERP evidence from covert tactile-spatial orienting. Psychophysiology 40:924-933. CrossRef Medline

Gallace A, Soto-Faraco S, Dalton P, Kreukniet B, Spence C (2008) Response requirements modulate tactile spatial congruency effects. Exp Brain Res 191:171-186. CrossRef Medline

Harrison SA, Tong F (2009) Decoding reveals the contents of visual working memory in early visual areas. Nature 458:632-635. CrossRef Medline

Heed T, Röder B (2010) Common anatomical and external coding for hands and feet in tactile attention: evidence from event-related potentials. J Cogn Neurosci 22:184-202. CrossRef Medline

Katus T, Andersen SK, Müller MM (2012) Maintenance of tactile shortterm memory for locations is mediated by spatial attention. Biol Psychol 89:39-46. CrossRef Medline

Katus T, Andersen SK, Müller MM (2014a) Common mechanisms of spatial attention in memory and perception: a tactile dual-task study. Cereb Cortex 24:707-718. CrossRef Medline

Katus T, Grubert A, Eimer M (2014b) Electrophysiological evidence for a sensory recruitment model of somatosensory working memory. Cereb Cortex. Advance online publication. Retrieved Aug. 20, 2014. CrossRef

Kostopoulos P, Albanese MC, Petrides M (2007) Ventrolateral prefrontal cortex and tactile memory disambiguation in the human brain. Proc Natl Acad Sci U S A 104:10223-10228. CrossRef Medline

Lepsien J, Nobre AC (2006) Cognitive control of attention in the human brain. Insights from orienting attention to mental representations. Brain Res 1105:20-31. CrossRef Medline

Miller J, Patterson T, Ulrich R (1998) Jackknife-based method for measuring LRP onset latency differences. Psychophysiology 35:99-115. CrossRef Medline

Nolan H, Whelan R, Reilly RB (2010) FASTER: fully automated statistical thresholding for EEG artifact rejection. J Neurosci Methods 192:152-162. CrossRef Medline

Pasternak T, Greenlee MW (2005) Working memory in primate sensory systems. Nat Rev Neurosci 6:97-107. CrossRef Medline

Postle BR (2006) Working memory as an emergent property of the mind and brain. Neuroscience 139:23-38. CrossRef Medline

Romo R, Salinas E (2003) Flutter discrimination: neural codes, perception, memory and decision making. Nat Rev Neurosci 4:203-218. CrossRef Medline

Soto-Faraco S, Azañón E (2013) Electrophysiological correlates of tactile remapping. Neuropsychologia 51:1584-1594. CrossRef Medline

Vogel EK, Machizawa MG (2004) Neural activity predicts individual differences in visual working memory capacity. Nature 428:748-751. CrossRef Medline

Yamamoto S, Kitazawa S (2001) Reversal of subjective temporal order due to arm crossing. Nat Neurosci 4:759-765. CrossRef Medline

Zhou YD, Fuster JM (1996) Mnemonic neuronal activity in somatosensory cortex. Proc Natl Acad Sci U S A 93:10533-10537. Medline 